\title{
CRESCIMENTO DE PLANTIOS CLONAIS DE EUCALIPTO NÃO DESBASTADOS NA REGIÃO DE MONTE DOURADO (PA $)^{1}$
}

Raul de Abreu Demolinari ${ }^{2}$, Carlos Pedro Boechat Soares ${ }^{3}$, Helio Garcia Leite $^{3}$ e Agostinho Lopes de Souza ${ }^{3}$

RESUMO - Este trabalho foi realizado com o objetivo de analisar o comportamento de variáveis de povoamento como área basal, volume, altura, diâmetro médio quadrático e número de árvores por hectare, ao longo do tempo. Para isso, foram utilizados dados de inventários florestais contínuos de plantações clonais de híbridos de Eucalyptus urophylla $x$ E. grandis não-desbastados, provenientes da empresa JARI CELULOSE S/A, no Estado do Pará. Foram selecionadas 63 parcelas permanentes, igualmente distribuídas em três classes de capacidade produtiva. Após as análises, verificou-se que as variáveis do povoamento possuíam relação direta com a capacidade produtiva do sítio ou local, apresentando diferentes taxas de crescimento ao longo do tempo; durante as idades iniciais, o número de árvores sobreviventes nos melhores sítios é maior do que nos piores. No entanto, com o passar do tempo há maior mortalidade nos melhores locais, invertendo esse comportamento.

Palavras-chave: Eucalyptus urophylla x Eucalyptus grandis, análise do crescimento e índice de local.

\section{GROWTH OF UNTHINNED CLONAL EUCALYPTUS PLANTATIONS IN THE REGION OF MONTE DOURADO (PA)}

\begin{abstract}
The objective of the present work was to analyze the behavior of stand variables such as basal area, volume, height and tree number per hectare over time. For this purpose, experiments used data from permanent forest inventory of unthinned clonal plantations of Eucalyptus grandis $x$ E. urophylla hybrids, from JARI CELULOSE, Pará, Brazil. A total of 63 permanent plots were selected and equally distributed in three productivity classes. The analysis confirmed that the stands variables showed direct relationship with the site, with different growth rates over time; the number of survivor trees is larger in sites with higher productivity in the first years after planting. However, in the best sites, there is a higher mortality with time, inverting this behavior.
\end{abstract}

Keywords: Eucalyptus urophylla $x$ Eucalyptus grandis, growth analysis and site index.

\section{INTRODUÇÃO}

Os projetos florestais, em especial aqueles referentes a plantios comerciais, são caracterizados pelo longo período de tempo entre os investimentos iniciais e os recebimentos futuros. Isso gera uma grande expectativa com relação ao volume total a ser colhido, uma vez que dele dependem as receitas a serem auferidas pelo empreendedor. Nesse sentido, a predição do crescimento e da produção dos povoamentos florestais é essencial para definir a quantidade de produtos que serão produzidos pela floresta, bem como para subsidiar as análises técnicas e econômicas dos projetos florestais (GUIMARÃES, 1994).

No Brasil, vários trabalhos foram realizados, objetivando ao estudo e modelagem do crescimento e da produção madeireira em florestas plantadas

\footnotetext{
${ }^{1}$ Recebido em 12.09.2006 e aceito para publicação em 07.04.2007.

${ }^{2}$ Engenheiro Florestal (M.Sc.). E-mail: <raul.abreu@aluminiocba.com.br>.

${ }^{3}$ Departamento de Engenharia Florestal da UFV. E-mail: <csoares@ufv.br>.
} 
(CAMPOS E TURNBULL, 1981; CAMPOS E RIBEIRO, 1983; TREVISOLJUNIOR, 1985; LEITE, 1990; SCOLFORO, 1990; ROSAS, 1994; GUIMARÃES, 1994; SOARES, 1998; NOGUEIRA, 2003; DIAS et al., 2005), dentre os quais se destaca a avaliação dos modelos do tipo povoamento total e de distribuição de diâmetros.

Embora a modelagem do crescimento e da produção florestal tenha avançado significativamente no Brasil, principalmente para plantios comerciais de pinus e eucalipto, percebe-se a necessidade de estudos sobre o crescimento de povoamentos comerciais, visando ao aprimoramento da modelagem do crescimento e da produção florestal, para proporcionar aos manejadores informações precisas sobre a época correta de colheita ou de intervenções como desbastes, além de informações sobre a adequação de espécies e, ou, procedências para determinadas localidades, entre outros.

Diante dessa constatação, percebe-se a necessidade de estudos sobre o crescimento de povoamentos comerciais, visando ao aprimoramento da modelagem do crescimento e produção florestal, para proporcionar aos manejadores informações precisas sobre a época correta de colheita ou de intervenções como desbastes, além de informações sobre a adequação de espécies e, ou, procedências para determinadas localidades, entre outros.

O objetivo deste trabalho foi, então, estudar o comportamento de variáveis do povoamento (área basal, altura, diâmetro quadrático, volume e número de árvores por hectare) ao longo do tempo.

\section{MATERIAL E MÉTODOS}

\subsection{Caracterização da área de estudo}

Este estudo foi realizado com dados de plantios clonais não-desbastados de híbridos de Eucalyptus urophylla $x$ Eucalyptus grandis, provenientes da empresa JARI CELULOSE S/A. cuja sede está situada na cidade de Monte Dourado, às margens do rio Jarí, no estado do Pará, Região Norte do Brasil.

Os plantios da empresa estão localizados em áreas cuja temperatura média anual é de aproximadamente $26^{\circ} \mathrm{C}$; a umidade relativa apresenta valores entre 80 e $85 \%$ em quase todos os meses do ano; a precipitação média anual está entre 2.000 e $2.500 \mathrm{~mm}$, com o período chuvoso compreendido entre os meses de dezembro e julho. Março é o mês mais chuvoso, enquanto os meses com menores índices pluviométricos compreendem-se entre julho e novembro, sendo outubro o mais seco; os solos predominantes da região são os Latossolos Amarelos (OLIVEIRA et al., 2004).

\subsection{Dados}

Os dados para a análise do crescimento foram obtidos de inventários florestais contínuos em plantios de eucalipto com espaçamento inicial entre plantas de $3 \times 3$ m e idades entre 24 e 84 meses. Foram selecionadas 63 parcelas permanentes com $500 \mathrm{~m}^{2}$ de área útil cada, com pelo menos cinco medições.

De posse das alturas totais médias das árvores dominantes $(H d)$ das parcelas, nas diferentes idades (I), foi realizada a classificação da capacidade produtiva dos plantios via Índices de Locais $(S)$, utilizando-se o Método da Curva-Guia (CLUTTER et al., 1983) e o modelo de Schumacher:

$\operatorname{LnHd}=\beta 0+\beta 1 / I+e_{i}$

em que: $\mathrm{Ln}=$ logaritmo neperiano; $\beta_{0}$ e $\beta_{1}=$ parâmetros dos modelos; $\mathrm{e}_{\mathrm{i}}=$ erro aleatório.

A equação ajustada foi avaliada através do coeficiente de determinação $\left(\mathrm{R}^{2}\right)$ e do coeficiente de variação (CV\%). A idade-índice adotada neste trabalho foi igual a 60 meses.

Para caracterizar tendências de crescimento dos povoamentos de eucalipto em volume e em área basal por hectare, em diferentes capacidades produtivas, para definir as idades técnicas de colheita (ITC) e as idades em que ocorreram as estagnações dos crescimentos em área basal por hectare, foram elaborados gráficos mostrando as curvas de produção e as curvas de incremento corrente mensal (ICM) e de incremento médio mensal (IMM). Para isso foi necessário ajustar o modelo de Clutter (1963), assim definido:

$\left\{\begin{array}{l}L n V_{2}=\beta_{0}+\beta_{1} / I_{2}+\beta_{2}\left(S_{1}\right)+\beta_{3}\left(\ln B_{2}\right)+e_{i} \\ L n B_{2}=\ln B_{1}\left(I_{1} / I_{2}\right)+\alpha_{1}\left(1-\left(I_{1} / I_{2}\right)\right)+\alpha_{2}\left(\left(1-\left(I_{1} / I_{2}\right)\right) S_{1}\right)+e_{i}\end{array}\right.$

em que: $I_{1}=$ idade atual, em meses; $I_{2}=$ idade futura, em meses; $S_{1}=$ índice de local, na idade $\mathrm{I}_{1} \mathrm{em} \mathrm{m} ; B_{1}$ = área basal inicial, $\mathrm{em} \mathrm{m}^{2} / \mathrm{ha} ; V_{2}=$ volume futuro $\mathrm{com}$ ou sem casca, em $\mathrm{m}^{3} / \mathrm{ha} ; B_{2}=$ área basal futura, em $\mathrm{m}^{2} / \mathrm{ha} ; \mathrm{ln}=$ logaritmo neperiano; $\beta_{0} \ldots \beta_{3}=$ parâmetros do modelo que projeta o volume; $\alpha_{0}$ e $\alpha_{1}=$ parâmetros do modelo que projeta a área basal; $\mathrm{e}_{\mathrm{i}}=$ erro aleatório. 
As equações ajustadas foram avaliadas através do coeficiente de determinação $\left(\mathrm{R}^{2}\right)$, do erro-padrão da estimativa $\left(\mathrm{S}_{\mathrm{y} . \mathrm{x}}\right)$, do coeficiente de variação (CV\%) e da análise gráfica da disperssão dos valores observados em relação aos estimados pelas equações.

As tendências de crescimento das variáveis do povoamento, altura total média $(H t)$, altura média das árvores dominantes $(H d)$, diâmetro médio ou quadrático $(q)$ e número de árvores por hectare $(\mathrm{N})$, em diferentes sítios, foram caracterizadas através de gráficos mostrando o comportamento das variáveis do povoamento ao longo do tempo. Além disso, foram elaborados gráficos de incremento corrente mensal (ICM) para verificar a estagnação do crescimento dessas variáveis. Para elaboração desses gráficos, foi necessário o ajuste de equações referentes ao modelo apresentado por Pienaar e Schiver (1981), originalmente proposto para descrever a sobrevivência de árvores. Esse ajuste é dado por:

$$
Y_{2}=Y_{1} \cdot \exp \left[-\beta_{0}\left(I_{2}^{\beta 1}-I_{1}^{\beta 1}\right)\right]+\mathrm{e}_{\mathrm{i}},
$$

em que: $Y_{2}=$ estimativa da variável do povoamento em uma idade futura; $Y_{1}=$ estimativa da variável do povoamento em uma idade atual; $I_{1}=$ idade atual, em meses; $I_{2}=$ idade futura, em meses; $\beta_{0}$ e $\beta_{1}=$ parâmetros do modelo; $\mathrm{e} \mathrm{e}_{\mathrm{i}}=$ erro aleatório.

As equações ajustadas foram avaliadas pelo coeficiente de correlação entre os valores observados e estimados ao quadrado $R y \hat{y}^{2}$; erro-padrão da estimativa $\left(\mathrm{S}_{\mathrm{y} . \mathrm{x}}\right)$; coeficiente de variação $(\mathrm{CV} \%)$ e análise gráfica da disperssão dos valores observados em relação aos estimados pelas equações.

\section{RESULTADOS E DISCUSSÃO}

\subsection{Classificação da capacidade produtiva}

O modelo de Schumacher, escolhido para a classificação dos índices de local $(S)$, ajustou-se bem aos dados, haja vista as estimativas do coeficiente de determinação $\left(\mathrm{R}^{2}\right)$ e do coeficiente de variação (CV\%).

$$
\begin{aligned}
& \text { LnHd }=3,510492274-14,88011596 I^{-1} \\
& \mathrm{R}^{2}=49,95 \% ; \mathrm{CV} \%=9,68 \%
\end{aligned}
$$

De acordo com as curvas de índice de local $(S)$, apresentadas na Figura 1, foram estabelecidas as seguintes classes de produtividade (Tabela 1 ).
Tabela 1 - Classificação de sítios para os plantios de Eucalyptus urophylla $x$ E. grandis

Table 1 - Site classification for Eucalyptus urophylla $x E$. grandis plantations

\begin{tabular}{ccc}
\hline $\begin{array}{c}\text { Classe de } \\
\text { Produtividade }\end{array}$ & $\begin{array}{c}\text { Amplitude } \\
\text { da Classe }(\mathrm{m})\end{array}$ & $\begin{array}{c}\text { Centro } \\
\text { de Classe }\end{array}$ \\
\hline Alta & $29 \leq S<35$ & $S=32$ \\
Média & $23 \leq S<29$ & $S=26$ \\
Baixa & $17 \leq S<23$ & $S=20$ \\
\hline
\end{tabular}

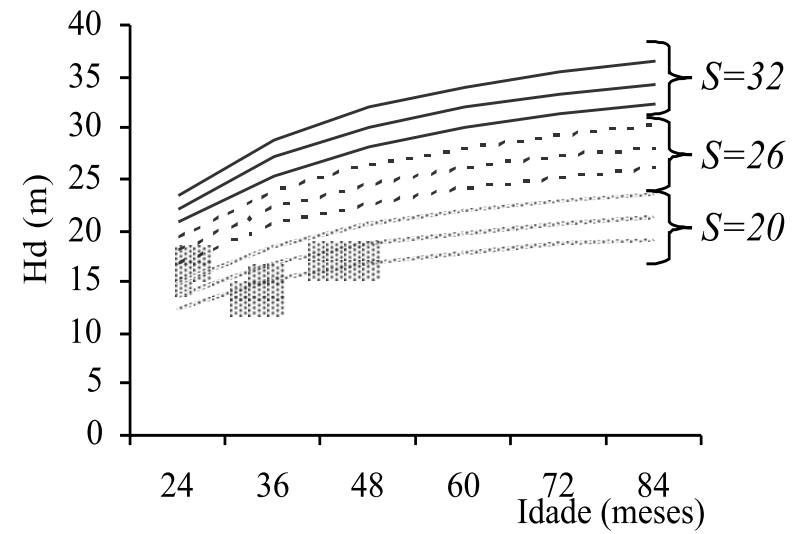

Figura 1 - Curvas de índice de local.

Figure 1 - Site index curves.

\subsection{Análise dos parâmetros populacionais}

\subsubsection{Volume e área basal por hectare}

O modelo de Clutter (1963) ajustou-se bem aos dados observados, haja vista as medidas de precisão das equações que projetam o volume com e sem casca por hectare e a área basal por hectare, apresentadas a seguir:

$\operatorname{LnVtcc}_{2}=2,1656-23,5127\left(1 / I_{2}\right)+0,0157\left(S_{1}\right)+1,0704\left(\operatorname{LnB} B_{2}\right)$ $\mathrm{R}^{2}=0,9824 ; \mathrm{S}_{\mathrm{y} \cdot \mathrm{x}}= \pm 0,0628 \ln \left(\mathrm{m}^{3} / \mathrm{ha}\right) ; \mathrm{CV}= \pm 4,86 \%$ LnVtsc $_{2}=1,7793-23,3495\left(1 / I_{2}\right)+0,0195\left(S_{1}\right)+1,1169\left(\operatorname{LnB}{ }_{2}\right)$ $\mathrm{R}^{2}=0,9836 ; \mathrm{S}_{\mathrm{y} . \mathrm{x}}= \pm 0,0654 \ln \left(\mathrm{m}^{3} / \mathrm{ha}\right) ; \mathrm{CV}= \pm 9,00 \%$ $\operatorname{Ln} B_{2}=\operatorname{Ln} B_{1}\left(I_{1} / I_{2}\right)+1,2614\left(1-\left(I_{1} / I_{2}\right)\right)+0,0696\left(1-\left(I_{1} / I_{2}\right)\right) S_{1}$ $\mathrm{R}^{2}=0,9667 ; \mathrm{S}_{\mathrm{y} . \mathrm{x}}= \pm 0,0628 \ln \left(\mathrm{m}^{2} / \mathrm{ha}\right) ; \mathrm{CV}= \pm 6,08 \%$

Contudo, verificou-se uma leve tendência de subestimação dos maiores volumes sem casca por hectare e área basal por hectare, bem como uma leve tendência de superestimação dos maiores volumes com casca (Figura 2).

R. Árvore, Viçosa-MG, v.31, n.3, p.503-512, 2007 
(a)

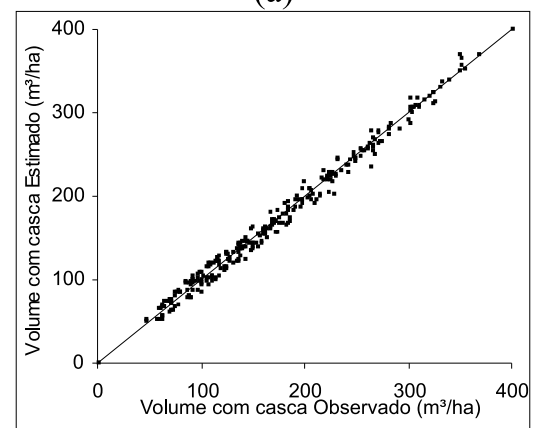

(b)

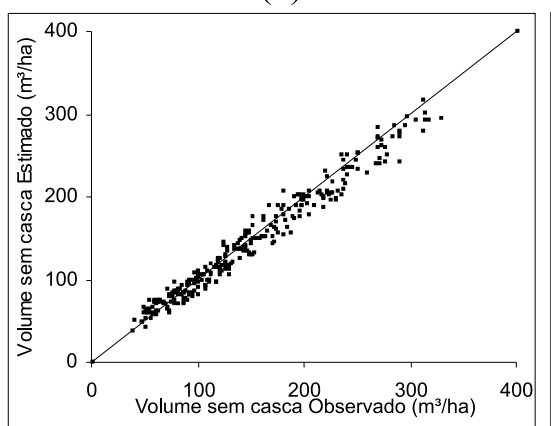

(c)

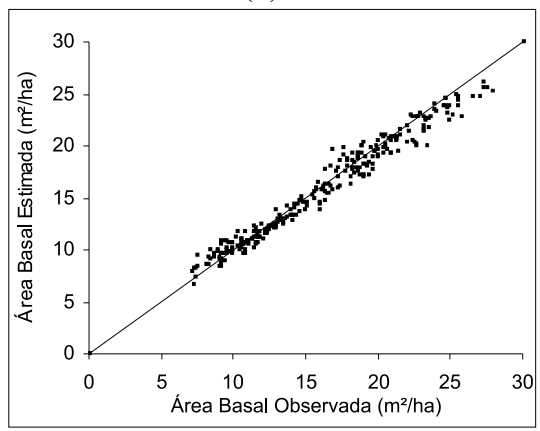

Figura 2 - Relação entre valores observados e estimados do volume com casca por hectare (a), volume sem casca por hectare (b) e área basal por hectare (c).

Figure 2 -Ratio between observed and estimated values for volume outside bark per hectare (a) the volume inside bark per hectare (b); and basal area per hectare.

De acordo com o Quadro 2 e as Figuras 3 e 4, verifica-se, que quanto maior a capacidade produtiva do local, menor a idade técnica de colheita. Estes resultados estão de acordo com Clutter et al. (1983), Campos et al. (1996), Soares (1998) e Dias et al. (2005). Cabe destacar a reduzida idade técnica de colheita em todos os sítios e a pequena diferença entre as idades técnicas de colheita dos volumes com e sem casca por hectare. Considerando que as idades técnicas de colheita foram obtidas com equações distintas e que estas possuem diferentes precisões, pode-se considerar que as ITCs foram iguais.

Para a análise do comportamento da área basal por hectare ao longo do tempo, nas três classes de índice de local $(S)$ foi necessário projetar a área basal para idades superiores e inferiores a 24 meses (Figura 5a). Isso foi necessário devido ao fato de o comportamento das curvas dos incrementos médios mensais (IMM), nos locais de maior produtividade ( $\mathrm{S}=32$ e $\mathrm{S}=26$ ), terem apresentado pontos de máximo aos 19 e 22 meses, (Figura 5b).

Quadro 2 - Idades técnicas de colheita (ITCs) dos volumes com e sem casca por hectare, considerando-se três classes de produtividade

Table 2 -Technical cutting age (ITC's) for the volumes outside and inside bark per hectare, considering three classes of productivity

\begin{tabular}{ccc}
\hline Indice de Local (S) & \multicolumn{2}{c}{ ITC (meses) } \\
\cline { 2 - 3 } & Vtcc & Vtsc \\
\hline 32 & 44 & 46 \\
26 & 50 & 52 \\
20 & 56 & 54 \\
\hline
\end{tabular}

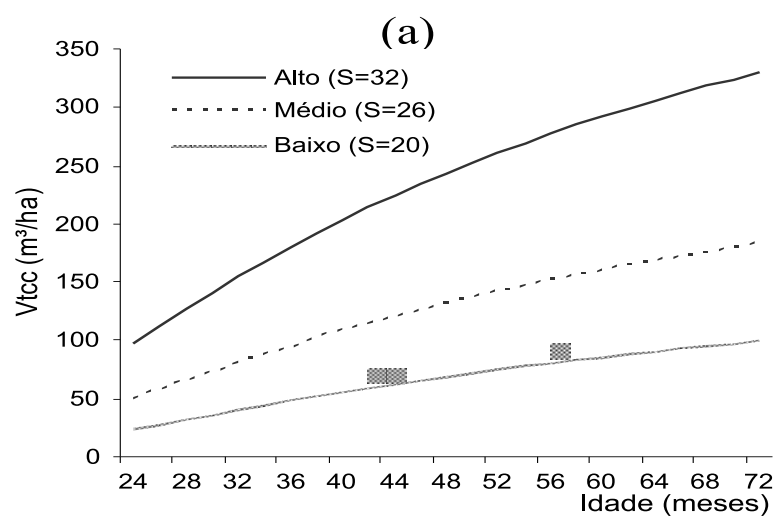

(b)

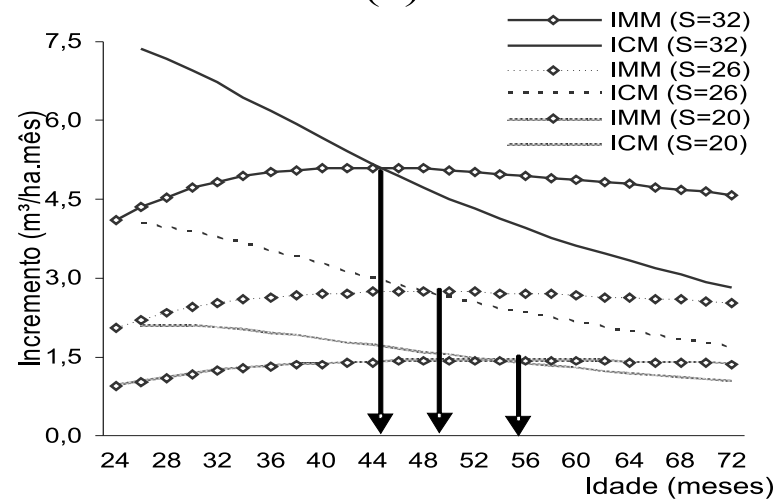

Figura 3 - Curvas de produção do volume com casca (Vtcc) por hectare (a) e curvas de incremento médio (IMM) e corrente (ICM) mensais (b), em três classes de produtividade.

Figure 3 - Production curves for outside bark volume (Vtcc) per hectare (a); curves of mean monthly increment (IMM) and current monthly increment (ICM) (b), in three classes of productivity. 

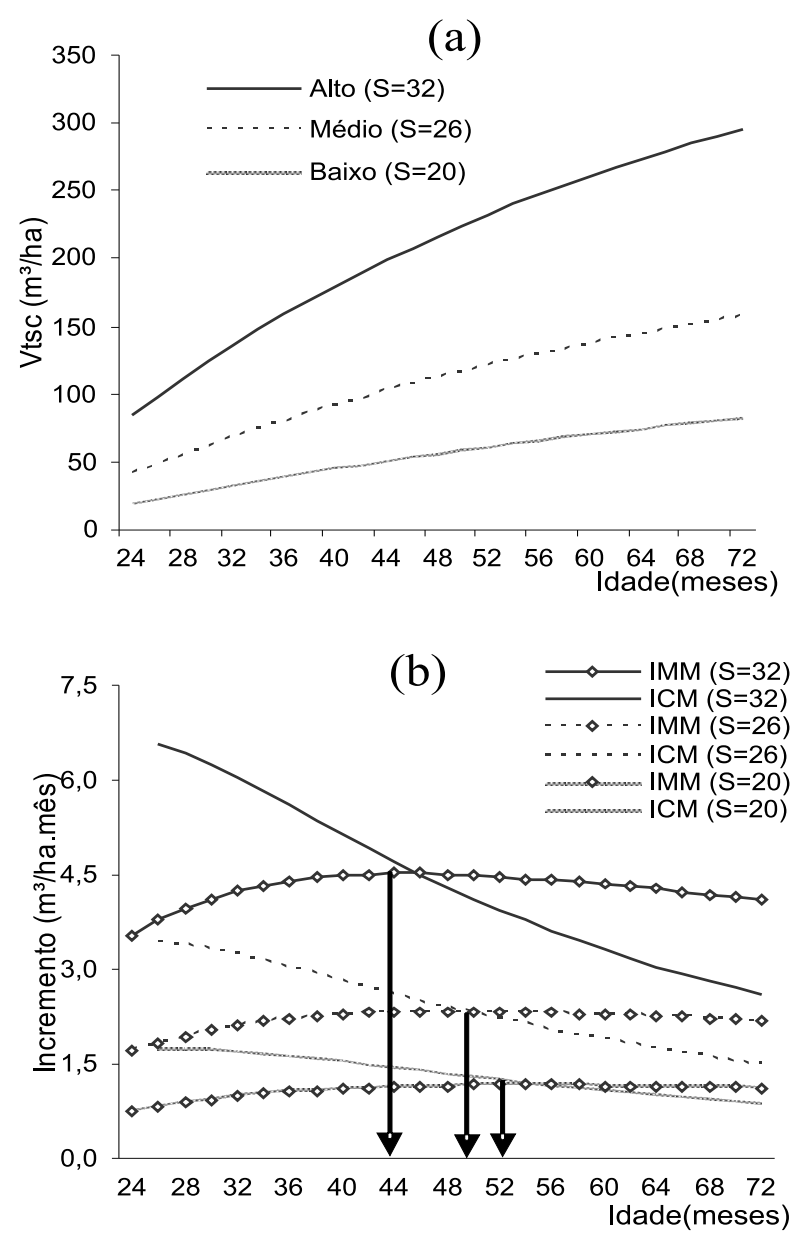

Figura 4 - Curvas de produção do volume sem casca (Vtsc) por hectare (a) e (b) curvas de incremento médio (IMM) e corrente (ICM) mensais, em três classes de produtividade.

Figure 4 - Production curves for inside bark volume (Vtcc) per hectare (a); curves of mean monthly increment $(I M M)$ and current monthly increment $(I C M)(b)$, in three classes of productivity.

\subsubsection{Altura dominante (Hd) e altura total (Ht)}

As equações ajustaram-se bem aos dados de altura dominante $(H d)$ e altura total $(H t)$, haja vista as medidas de precisão (Quadro 2) e os gráficos de dispersão (Figuras 6 e 7).

Analisando as curvas de crescimento em altura (Figura 8-a e 9-a), verificou-se o aumento da altura com a idade (CALEGÁRIO et al., 2005; FLORIANO et al., 2006; TONINI et al., 2006), bem como uma relação direta entre a altura das árvores e a capacidade produtiva
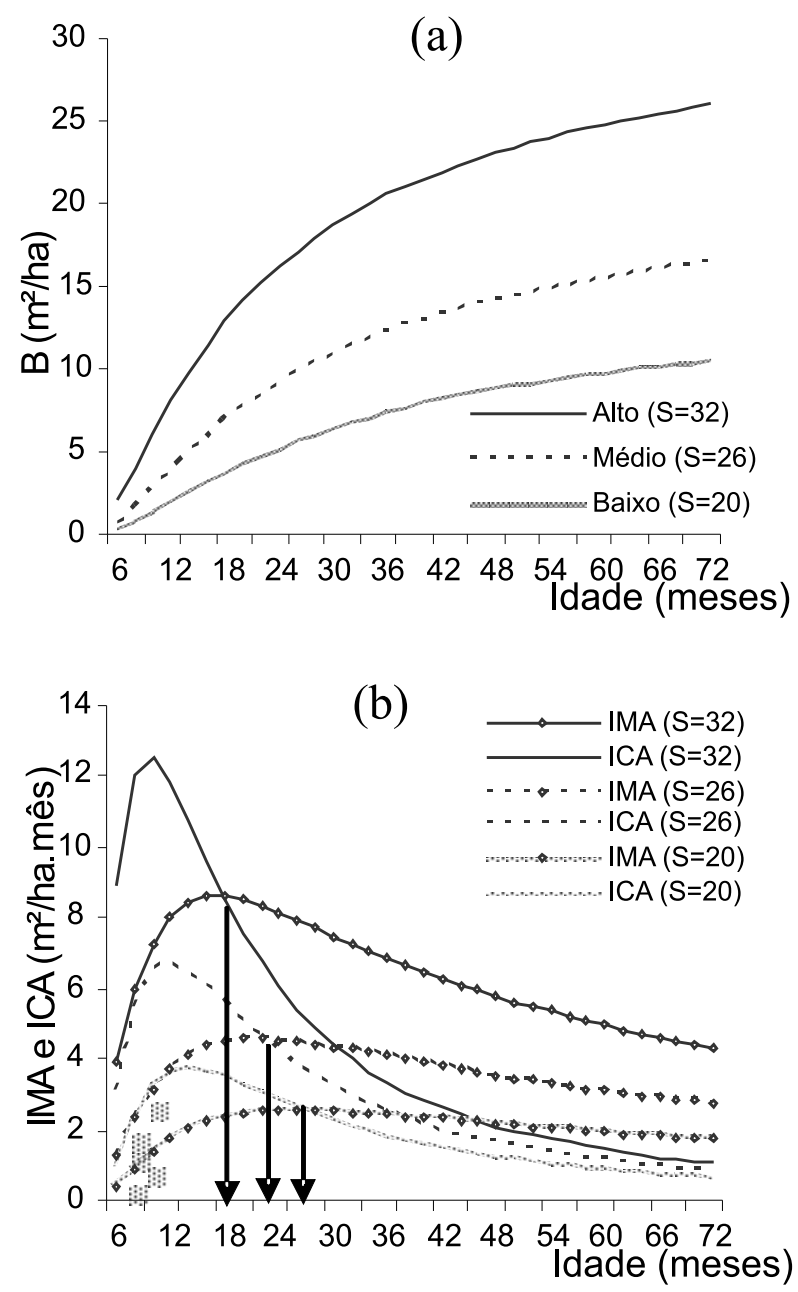

Figura 5 - Curvas de produção em área basal por hectare (a) e curvas de incremento médio (IMM) e corrente (ICM) mensais, em três classes de produtividade.

Figure 5 - Production curves in basal área per hectare (a); curves of mean monthly increment (IMM) and current monthly increment (ICM) - (b), in three classes of productivity.

do local (ASSMANN, 1970). Com relação às curvas dos incrementos correntes mensais (Figuras 8be 9b), observaramse incrementos decrescentes a partir dos 24 meses de idade, tanto para a altura média das árvores dominantes quanto para a altura total média do povoamento. Cabe destacar, no entanto, que para o índice de local $(S)$ igual a 32 há um decréscimo maior, de tal forma que as curvas dos incrementos correntes dos locais com índices iguais a 32 e 26 se igualam ou se cruzam. Isso se deve, possivelmente, à estagnação do crescimento, que ocorre primeiro em locais de maior produtividade.

R. Árvore, Viçosa-MG, v.31, n.3, p.503-512, 2007 
Quadro 3 - Equações ajustadas de altura dominante $(H d)$ e altura total $(H t)$, considerando-se três classes de produtividade e respectivas medidas de precisão

Table 3 - Adjusted equations for dominant height $(\mathrm{Hd})$ and total height $(\mathrm{Ht})$, considering three classes of productivity and their respective precision measures

\begin{tabular}{ccccc}
\hline Produtividade & Equações & $\left(r_{y \hat{y}}\right)^{2}$ & $\mathrm{~S}_{\mathrm{y} . \mathrm{x}}(\mathrm{m})$ & $\mathrm{CV} \%$ \\
\hline Baixa & $H d_{2}=H d_{1} \exp \left(-3,100634\left(I_{2}^{-0,376416}-I_{1}^{-0,376416}\right)\right)$ & $73,61 \%$ & 1,1125 & 5,34 \\
$(\mathrm{~S}=20)$ & $H t_{2}=H t_{1} \exp \left(-5,536091\left(I_{2}^{-0,115749}-I_{1}^{-0,115749}\right)\right)$ & $96,71 \%$ & 0,3802 & 2,17 \\
\hline Média & $H d_{2}=H d_{1} \exp \left(-4,733260\left(I_{2}^{-0,437713}-I_{1}^{-0,437713}\right)\right)$ & $87,25 \%$ & 1,1649 & 4,59 \\
$(\mathrm{~S}=26)$ & $H t_{2}=H t_{1} \exp \left(-4,745588\left(I_{2}^{-0,247332}-I_{1}^{-0,247332}\right)\right)$ & $98,63 \%$ & 0,3460 & 1,66 \\
\hline Alta & $H d_{2}=H d_{1} \exp \left(-6,506210\left(I_{2}^{-0,625710}-I_{1}^{-0,625710}\right)\right)$ & $86,33 \%$ & 1,2279 & 4,18 \\
$(\mathrm{~S}=32)$ & $H t_{2}=H t_{1} \exp \left(-4,932926\left(I_{2}^{-0,410185}-I_{1}^{-0,410185}\right)\right)$ & $98,43 \%$ & 0,3352 & 1,44 \\
\hline
\end{tabular}

(a)

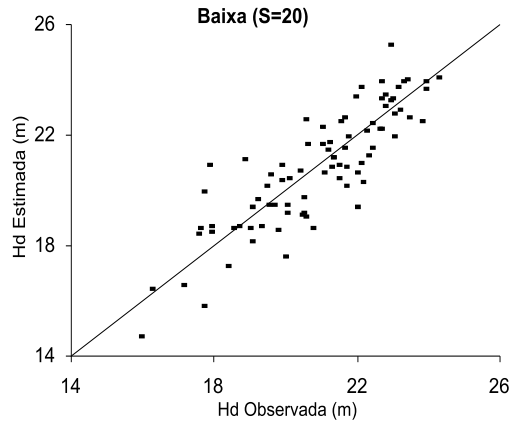

(b)

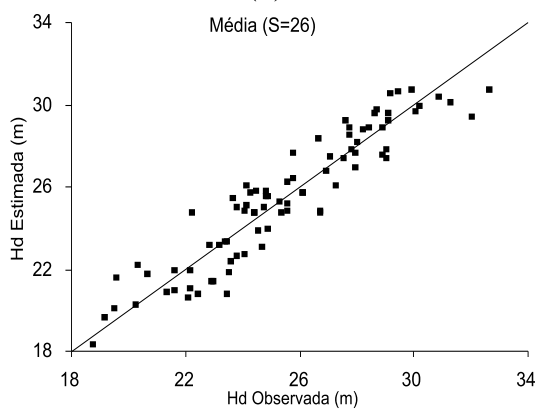

(c)

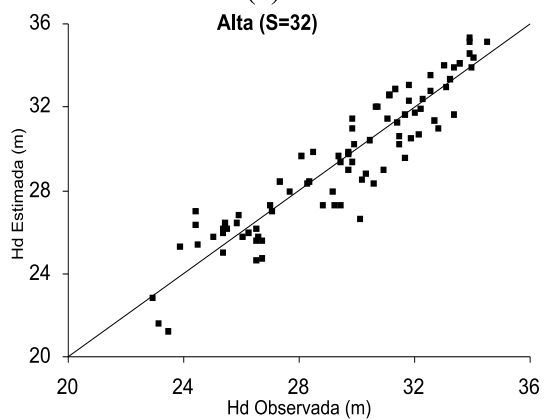

Figura 6 - Relação entre alturas dominantes observadas e estimadas, considerando-se três classes de produtividade. Figure 6 - Ratio between observed and estimated dominant heights considering three classes of productivity.

(a)

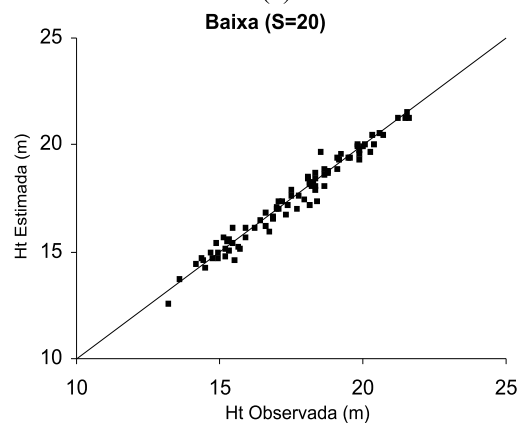

(b)

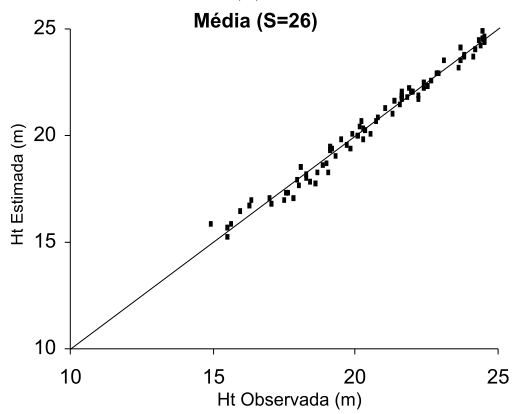

(c)

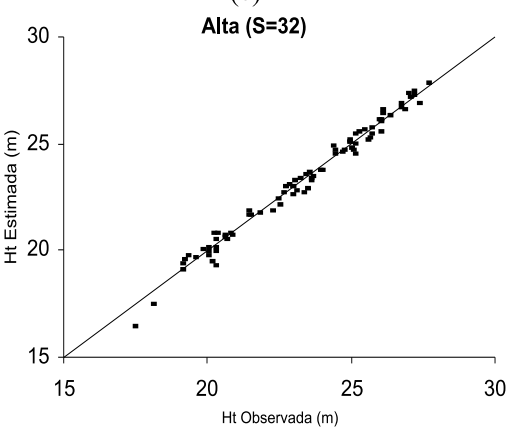

Figura 7 - Relação entre alturas totais observadas e estimadas, considerando-se três classes de produtividade. Figure 7 - Ratio between observed and estimated total heights considering three classes of productivity.

\subsubsection{Diâmetro médio ou quadrático (q) e número de árvores por hectare $(\mathbf{N})$}

As equações ajustaram-se bem aos dados observados (Quadro 4 e Figuras 10 e 11), com coeficientes de correlação entre os valores observados e estimados ao quadrado maiores que $89 \%$.
Na Figura 12-a, pode-se observar que as curvas de crescimento em diâmetro, nos locais com índices $(S)$ iguais a 32 e 26, apresentaram-se praticamente paralelas, ou seja, com tendências de crescimento semelhantes (SCHEEREN et al., 2004). Quanto ao local com índice $(S)$ igual a 20 (baixa produtividade), verificaram-se taxas

R. Árvore, Viçosa-MG, v.31, n.3, p.503-512, 2007 
menores de crescimento. Ainda na Figura 12a, observase que as curvas de crescimento em diâmetro não atingiram um valor assintótico aos 72 meses de idade, indicando não haver estagnação do crescimento em diâmetro. Contudo, se árvores menores morrem nas menores classes diamétricas, o valor do diâmetro médio ou quadrático $(q)$ tende sempre a aumentar com a idade, mesmo que o povoamento apresente estagnação do crescimento volumétrico, por exemplo.
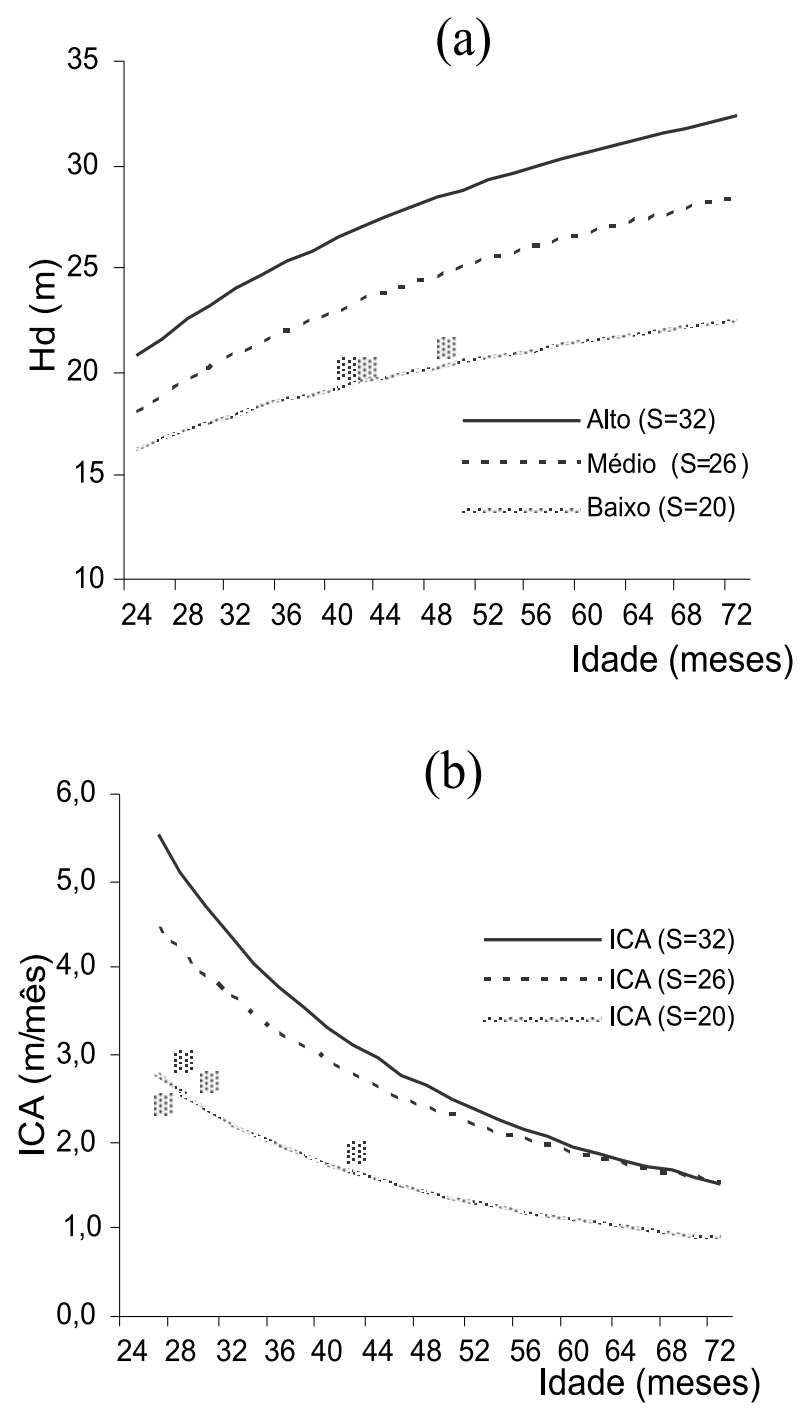

Figuras 8 - Curvas de altura dominante (Hd) - (a) e curvas de incremento corrente mensal (ICM) - (b), considerando-se três classes de produtividade.

Figure 8 -Dominant height curves $(H d)-(a)$; current monthly increment curves $(I C M)-(b)$, considering three classes of productivity.
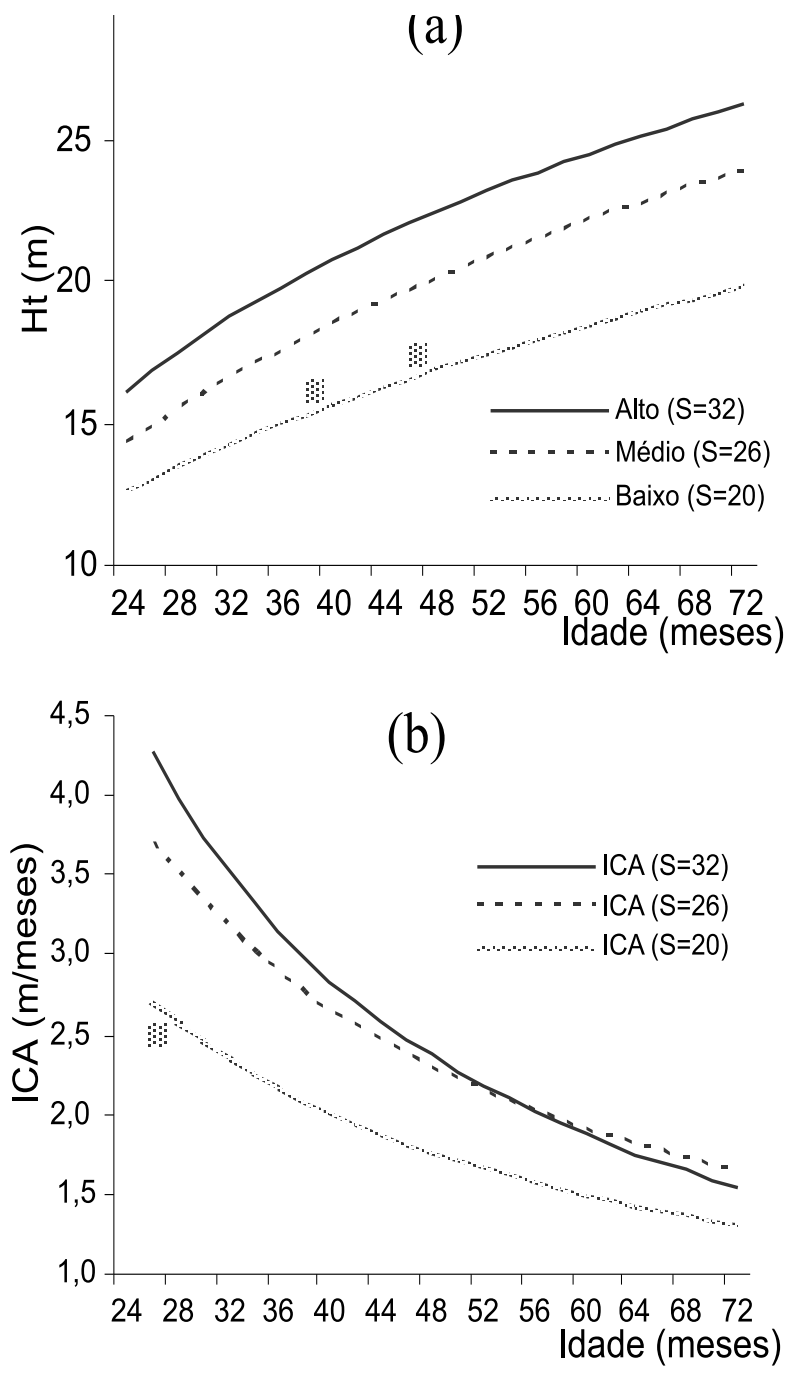

Figuras 9 - Curvas de altura total (Ht) - (a) e curvas de incremento corrente mensal (ICM) (b), considerando-se diferentes classes de índice de local.

Figure 9-Total height curves $(\mathrm{Ht})-(a)$ and current monthly increment curves $(I C M)-(b)$, considering three classes of productivity.

Em idades iniciais, o número de árvores por hectare é maior no local de maior capacidade produtiva $(S=32)$ (Figura 13). Contudo, esse comportamento se inverte com o passar do tempo. Isso se deve à mortalidade que se inicia mais cedo em locais de maior capacidade produtiva, causando a redução do número de árvores. Comportamento semelhante foi observado por Machado (1979), em estudo de sobrevivência em plantios homogêneos de Pinus taeda.

R. Árvore, Viçosa-MG, v.31, n.3, p.503-512, 2007 
Quadro 4 - Equações ajustadas do diâmetro médio ou quadrático $(q)$ e número de árvores por hectare $(N)$, considerandose as três classes de produtividade e respectivas medidas de precisão

Table 4 -Adjusted equations for mean or quadratic diameter $(q)$ and number of trees per hectare $(N)$, considering three classes of productivity and their respective precision measures

\begin{tabular}{ccccc}
\hline Produtividade & Equações & $\left(r_{y \hat{y}}\right)^{2}$ & $\mathrm{~S}_{\mathrm{y} . \mathrm{x}}$ & $\mathrm{CV} \%$ \\
\hline Baixa & $q_{2}=q_{1} \exp \left(-130,8112\left(I_{2}{ }^{-0,002306}-I_{1}^{-0,002306}\right)\right)$ & $96,21 \%$ & 0,2291 & 1,97 \\
$(\mathrm{~S}=20)$ & $N_{2}=N_{1} \exp \left(-0,000151\left(I_{2}{ }^{1,426782}-I_{1}^{1,426782}\right)\right)$ & $94,95 \%$ & 26,5 & 2,68 \\
\hline Média & $q_{2}=q_{1} \exp \left(-403,7016\left(I_{2}{ }^{-0,000853}-I_{1}^{-0,000853}\right)\right)$ & $98,23 \%$ & 0,2354 & 1,65 \\
$(\mathrm{~S}=26)$ & $N_{2}=N_{1} \exp \left(-0,000130\left(I_{2}{ }^{1,507597}-I_{1}^{1,507597}\right)\right)$ & $92,73 \%$ & 27,2 & 2,69 \\
\hline Alta & $q_{2}=q_{1} \exp \left(-247,9137\left(I_{2}{ }^{-0,001262}-I_{1}^{-0,001262}\right)\right)$ & $96,47 \%$ & 0,2805 & 1,75 \\
$(\mathrm{~S}=32)$ & $N_{2}=N_{1} \exp \left(-0,000265\left(I_{2}{ }^{1,507136}-I_{1}^{1,507136}\right)\right)$ & $89,29 \%$ & 30,9 & 3,07 \\
\hline
\end{tabular}

(a)

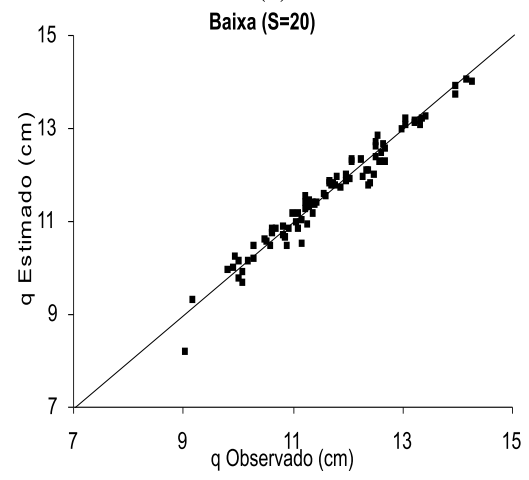

(b)

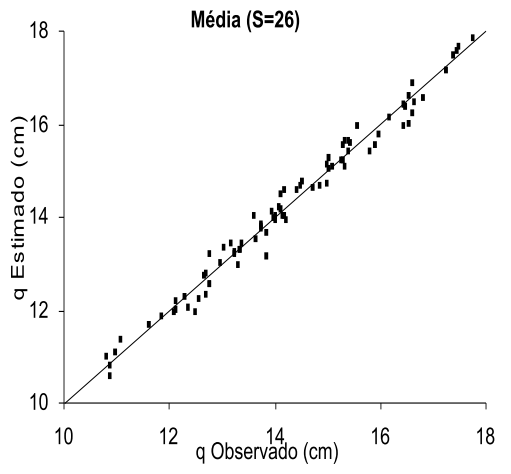

(c)

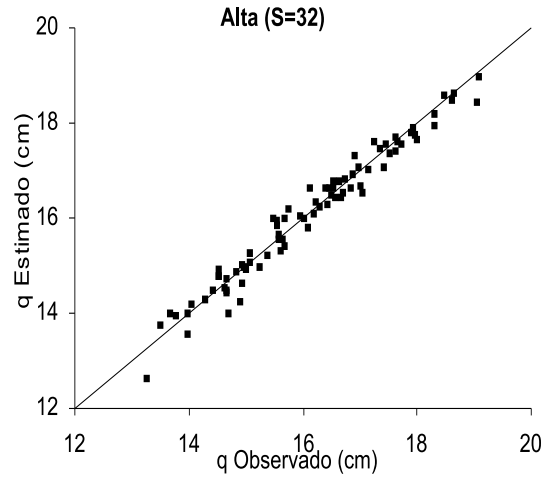

Figura 10 - Relação entre valores observados e estimados de diâmetro médio ou quadrático, considerando-se três classes de produtividade.

Figure 10-Ratio between observed and estimated values for mean or quadratic diameter considering three classes of productivity.

(a)

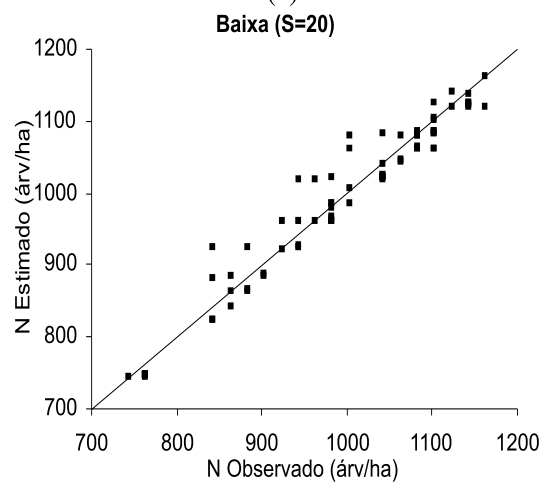

(b)

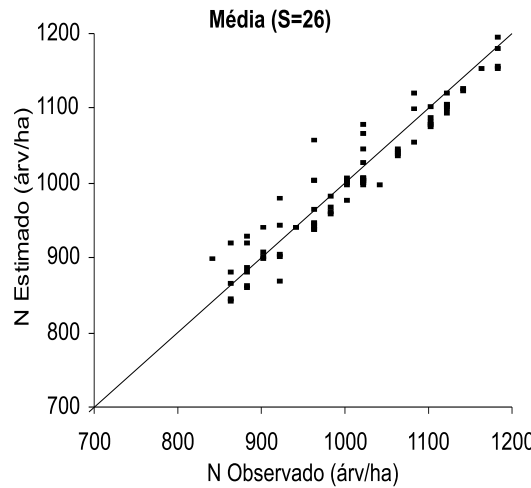

(c)

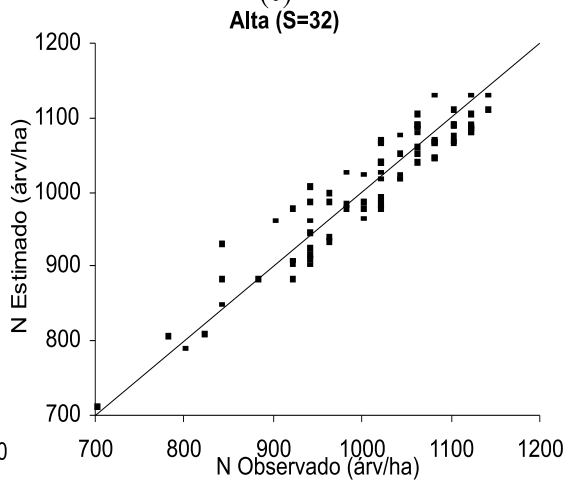

Figura 11 - Relação entre valores observados e estimados do número de árvores por hectare, considerando-se três classes de produtividade.

Figure 11 -Ratio between observed and estimated values for number of trees per hectare considering three classes of productivity.

R. Árvore, Viçosa-MG, v.31, n.3, p.503-512, 2007 

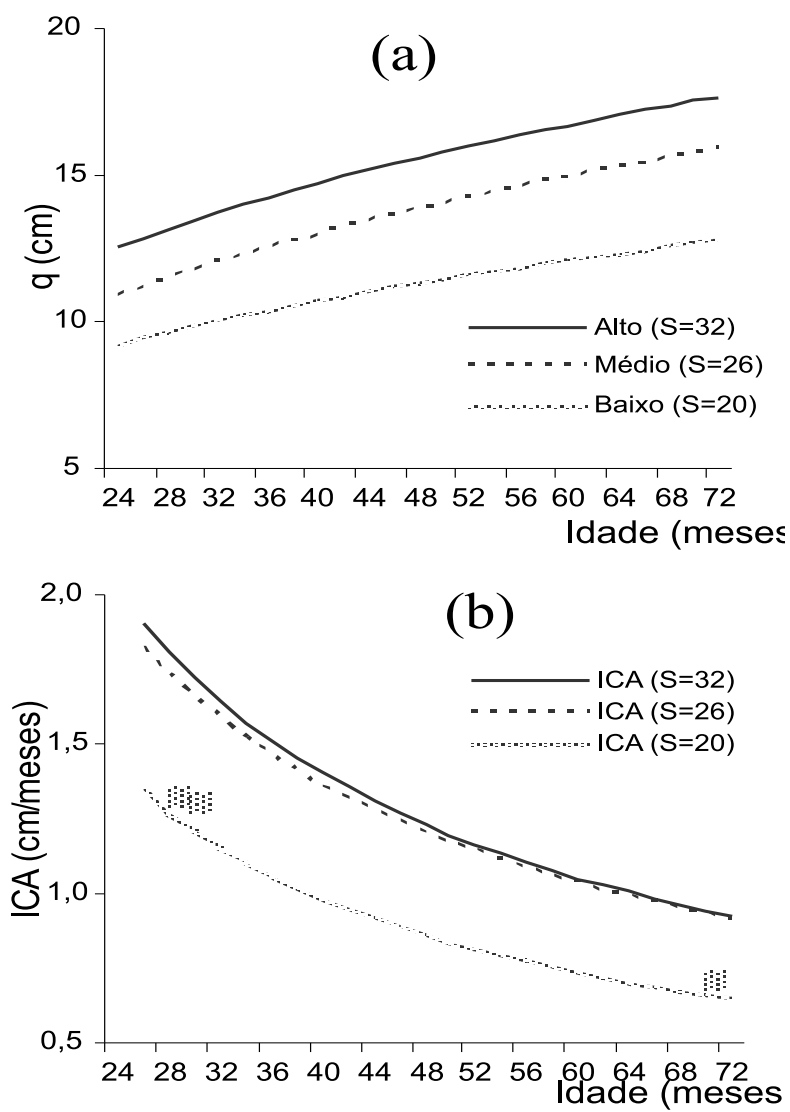

Figuras 12-Curvas de crescimento do diâmetro médio ou quadrático (a) e curvas dos incrementos correntes mensais (ICM)

(b), considerando-se três classes de produtividade.

Figure 12 - Growth curves for mean or quadratic diameter

(a) and current monthly increment curves (ICM)

- (b), considering three classes of productivity.

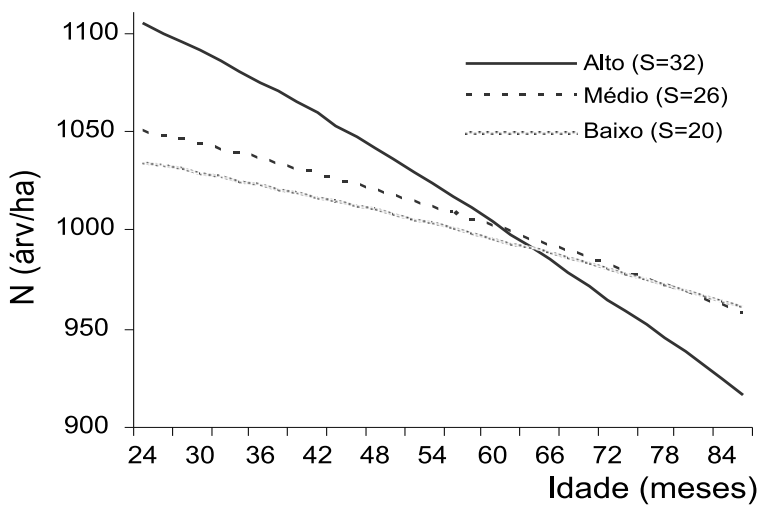

Figura 13 - Número de árvores sobreviventes por hectare ao longo do tempo, considerando-se três classes de produtividade.

Figure 13 - Number of survivor trees per hectare over time, considering three classes of productivity.

\section{CONCLUSÕES}

Após a realização deste estudo, pôde-se concluir que:

1) As variáveis do povoamento possuem relação direta com a capacidade produtiva do sítio, apresentando diferentes taxas de crescimento ao longo do tempo.

2) Nos primeiros anos após o plantio, a sobrevivência das árvores é maior nos melhores sítios. No entanto, essa tendência se inverteu com o passar do tempo.

\section{REFEÊNCIAS}

ASSAMANN, E. The principles of Forest yield study. New York: Pergamon Press, 1970. 506p.

CALEGÁRIO, N. et al.Estimativa do crescimento de povoamentos de Eucalyptus baseada na teoria dos modelos não lineares em multinível de efeito misto.Ciência Florestal, v.15, n.3, p.285-292, 2005.

CAMPOS, J. C. C.; RIBEIRO, J. C. A análise de tronco como fonte de dados para estudos de crescimento florestal. Revista Árvore, v.11, n.1, p.66-77, 1987.

CAMPOS, J. C. C.; ROSAS, M. P.; LEITE, H. G. Comparação de alternativas de determinação da idade técnica de corte em plantações de eucalipto. Revista Árvore, v.20, n.1, p.37-49, 1996.

CAMPOS, J. C. C.; TURNBULL, K. J. Um sistema para estimar produção por classe de diâmetro e sua aplicação na interpretação de efeitos de desbaste. Revista Árvore, v.5, n.1 p.1-16, 1981.

CLUTTER, J. L. Compatible growth and yield models for lobolly pine. Forest Science, v.9, n.3, p.354-371, 1963.

CLUTTER, J. L. et al. Timber management: A quantitative approach. New York: John Wiley \& Sons, 1983. 333p.

DIAS A. N. et al. Emprego de um modelo de crescimento e produção em povoamentos desbastados de eucalipto. Revista Árvore, v.29, n.5, p.731-739, 2005.

FLORIANO, E. P.et al. Ajuste e seleção de modelos tradicionais para série temporal de dados de altura das árvores. Ciência Florestal, v.16, n.2, p.177-199, 2006.

R. Árvore, Viçosa-MG, v.31, n.3, p.503-512, 2007 
GUIMARÃES, D. P. Desenvolvimento de um modelo de distribuição diamétrica de passo invariante para prognose e projeção da estrutura de povoamentos de eucalipto. 1994. 178f. Tese (Doutorado em Ciência Florestal) - Universidade Federal de Viçosa, Viçosa, MG, 1994.

LEITE, H. G. Ajuste de um modelo de estimação de freqüência e produção por classe de diâmetro, para povoamentos de Eucalyptus saligna Smith. 1990. 97f. Dissertação (Mestrado em Ciência Florestal) - Universidade Federal de Viçosa, Viçosa, MG, 1990.

MACHADO, S. A. Estimativa de sobrevivência de Pinus taeda em plantios homogêneos. Revista Floresta, v.10, n.1, p.73-76, 1979.

NOGUEIRA, G. S. Modelagem do crescimento e da produção de povoamentos de Eucalyptus sp. e de Tectona grandis submetidos a desbaste. 2003. 132f. Tese (Doutorado em Ciência Florestal) - Universidade Federal de Viçosa, Viçosa, MG, 2003.

OLIVEIRA, L. L. et al. Mapas dos parâmetros climatológicos do estado do Pará: umidade, temperatura e insolação, médias anuais. In: CONGRESSO BRASILEIRODE

METEREOLOGIA, 13., 2004,Fortaleza. Anais... Fortaleza: 2004. 7p.

PIENAAR, L. V.; SHIVER, B. D. Survival functions for site prepared slash pine plantations in the flat woods of Georgia Northern Florida. Southern Journal Forestry, v.5, n.2, p.59-62, 1981.
ROSAS, M. P. Alternativas de determinação da idade técnica de corte de Eucalyptus urophylla. 1994. 85f. Dissertação (Mestrado em Ciência Florestal) Universidade Federal de Viçosa, Viçosa, MG, 1994.

SCHEEREN, L. W.; SCHNEIDER, P. R.; FINGER, C. A. G. Crescimento e produção de povoamentos monoclonais de Eucalyptus saligna Smith manejados com desbaste, na região sudeste do Estado do Rio Grande do Sul. Ciência Florestal, v.14, n.2, p.111-122, 2004.

SCOLFORO, J. R. S. Sistema integrado para predição e análise presente e futura do crescimento e produção, com otimização de remuneração de capitais, para Pinus caribaea var. hondurensis. 1990. 308f. Tese (Doutorado em Ciência Florestal) Universidade Federal do Paraná, Curitiba, 1990.

SOARES, C. P. B. Um modelo para o gerenciamento da produção de madeira em plantios comerciais de eucalipto. 1998. 72f. Tese (Doutorado em Ciência Florestal) Universidade Federal de Viçosa, Viçosa, MG, 1998.

TONINI, H.; SCHNEIDER, P. R.; FINGER, C. A. G. Curvas de índice de local para povoamentos clonais de Eucalyptus saligna Smith para a Depressão Central e Serra do Sudeste, Rio Grande do Sul. Ciência Florestal, v.16, n.1, p. 27-43, 2006.

TREVISOL JUNIOR, T. L. Análise de um modelo compatível de crescimento e produção em plantações de Eucalyptus grandis W. Hill ex Maiden. 1985. 88f. Dissertação (Mestrado em Ciência Florestal) Universidade Federal de Viçosa, Viçosa, MG, 1985. 